\title{
Análise do ciclo de vida de veículos convencional, elétrico e híbrido plug-in para condições brasileiras
}

As emissões veiculares e o alto consumo de energia vinculado à cadeia de produção e uso de automóveis causam impactos significativos ao meio ambiente. Algumas medidas podem ser implementadas a fim de minimizar os danos causados por essas atividades. $O$ uso eficiente de energia, assim como escolhas por produtos e serviços que liberem menores quantidades de poluentes, são práticas que visam à diminuição dos impactos ao meio ambiente. Diante dessa realidade, veículos elétricos e híbridos tem atraído a atenção por parte de fabricantes, governos e consumidores, principalmente devido aos problemas relacionados à poluição atmosférica causada por emissões veiculares, aquecimento global e diminuição da utilização de combustíveis fósseis. O presente trabalho possui o objetivo de realizar uma Análise do Ciclo de Vida (ACV) para diferentes cenários da tecnologia veicular/combustível no Brasil, a fim de se conhecer os impactos ambientais causadas por cada um desses sistemas. Os resultados obtidos mostram que cenários que utilizam etanol como parte do combustível, possui o maior potencial de impacto para a acidificação. Já os sistemas que utilizam gasolina como combustível possuem maior potencial de impacto para o aquecimento global. Finalmente, os veículos híbridos e elétricos que utilizam a bateria de íon de lítio possuem maiores impactos para a toxicidade humana.

Palavras-chave: ACV, Veículo Convencional; Veículo Elétrico; Veículo Híbrido Plug-in.

\section{Life cycle assessment of conventional, electric, plug-in hybrid vehicles in brazilian conditions}

\begin{abstract}
Vehicle emissions and high energy consumption linked to the chain of automobile production and use have significant environmental impacts. Some measures can be implemented to minimize the damage caused by these activities. The efficient use of energy, as well as choices for products and services that release lower amounts of pollutants are practices that aim at reducing the environmental impacts. Given this reality, electric and hybrid vehicles has attracted the attention of manufacturers, governments and consumers, mainly due to problems related to air pollution caused by vehicle emissions, global warming and reducing the use of fossil fuels. This work has the objective to carry out a Life Cycle Assessment (LCA) for different scenarios of vehicle technology / fuel in Brazil, in order to find out the environmental impacts caused by each of these systems. The results show that scenarios using ethanol as part of the fuel, has the biggest potential impact to acidification. The Systems that use gas as fuel has the biggest potential impact to global warming. Finally, electric and hybrid vehicles using lithium-ion battery have major impacts on human toxicity.
\end{abstract}

Keywords: LCA; Conventional Vehicle; Electric Vehicle; Hybrid Vehicle Plug-in.

\section{Topic: Sustentabilidade nos Transportes}

Reviewed anonymously in the process of blind peer.

Lidiane La Picirelli Souza

Universidade Federal de Itajubá, Brasil

http://lattes.cnpq.br/0421086793728283

lidiane ehd@hotmail.com

Electo Eduardo Silva Lora

Universidade Politécnica de São Petersburgo, Brasil

http://lattes.cnpq.br/5479717772667720

silva.electo52@gmail.com

José Carlos Escobar Palacio

Universidade Federal de Itajubá, Brasil

http://lattes.cnpq.br/1472037977658347

jocescobar@gmail.com
Received: 20/03/2016

Approved: 24/08/2016

\author{
Mateus Henrique Rocha \\ Universidade Federal de Itajubá, Brasil \\ http://lattes.cnpq.br/0475301802380674 \\ mateus0@yahoo.com.br \\ Maria Luiza Grillo Renó \\ Universidade Federal de Itajubá, Brasil \\ http://lattes.cnpq.br/4411491026320331 \\ malureno@yahoo.com.br
}

Referencing this:

SOUZA, L. P.; LORA, E. E. S.; PALACIO, J. C. E.; ROCHA, M. H.; RENÓ, M. L. G.. Análise do ciclo de vida de veículos convencional, elétrico e híbrido plug-in para condições brasileiras. Revista Ibero-Americana de Ciências Ambientais, v.7, n.3, p.144-159, 2016. DOI:

http://doi.org/10.6008/SPC2179-6858.2016.003.0012 


\section{INTRODUÇÃO}

Nos países em desenvolvimento como a Índia, China e Brasil, o crescimento anual na frota de automóveis aumenta rapidamente, assim como as emissões ambientais vinculadas ao transporte. Nesses países o transporte público é insuficiente, a gestão de veículos é deficiente, além da tecnologia veicular ser obsoleta. Para agravar ainda mais a situação, o transporte não motorizado está sendo substituído por veículos motorizados, devido ao aumento de renda da população (XIMENES et al., 2008).

De acordo com o Ministério de Minas e Energia (EPE, 2011), o setor de transportes no Brasil consome entre 25 a 30\% da energia total consumida no país, sendo o segundo maior consumidor da energia total no país. Além disso, esse setor ainda é responsável por $55 \%$ do consumo de derivados de petróleo no Brasil. No entanto, se comparado com a média mundial, o consumo de combustíveis fósseis no setor de tranporte do Brasil é menor, devido à adição de $25 \%$ de etanol anidro a gasolina e ao grande número de veículos que utilizam o etanol hidratado como combustível veicular.

No entanto, mesmo sob essas condições, o processo da combustão emite gases como o dióxido de carbono $\left(\mathrm{CO}_{2}\right)$, hidrocarbonetos $(\mathrm{HC})$, óxidos de enxofre $\left(\mathrm{SO}_{\times}\right)$, monóxido de carbono $(\mathrm{CO})$ e óxidos de nitrogênio $\left(\mathrm{NO}_{\mathrm{x}}\right)$, entre outros. Esses gases poluentes dão origem a muitas situações prejudiciais ao meio ambiente, tais como a intensificação do efeito estufa, alterações climáticas, acidificação dos solos e dos recursos aquíferos, aparecimento de problemas respiratórios e cancerígenos nas populações que estão mais expostas a essas emissões, entre outros (MOMOH et al., 2009).

Segundo Balat et al. (2008) grande parte do esforço atual para controle das emissões de Gases do Efeito Estufa (GEE) e a busca pela sustentabilidade no setor automotivo, se concentra em: Reduzir o consumo de energia; Aumentar a eficiência da conversão ou da utilização de energia; Utilização de combustíveis alternativos, sobretudo os de base renovável; Desenvolver motores mais eficientes; Promover a evolução tecnológica de forma a implantar novos sistemas de propulsão; Introduzir medidas para o controle e minimização do tráfego; Incentivar novos hábitos de condução; Promover a captura e armazenamento de $\mathrm{CO}_{2}$.

Logo, pode-se constatar que existem possíveis soluções para problemas relacionados ao setor automotivo do país. Para que isso ocorra, será necessários grandes investimentos combinados com políticas de apoio, que são fundamentais para implementar e financiar mudanças. Em face dessa necessidade de mudança, existe uma técnica capaz de analisar toda a cadeia de produção, uso e fim de vida de processos e produtos, considerando os recursos energéticos, as matérias primas e emissões decorrentes dessas atividades. Esse método é a Análise do Ciclo de Vida (ACV) que neste trabalho foi aplicada em três sistemas: (1) veículo com motor de combustão interna, que utiliza a gasolina como fonte de energia (ICEVg); (2) veículo elétrico movido a eletricidade (BEV); (3) veículo híbrido plug-in movido a gasolina e eletricidade (PHEV).

Por fim, justifica-se a realização deste trabalho, diante da necessidade de estudos adequados que gerem informações relevantes e evidenciem os possíveis impactos ambientais causados por sistemas 
automotivos, para que se possam elaborar ferramentas capazes de auxiliar nas tomadas de decisões relacionadas com as melhorias das condições sociais e reduções dos impactos ambientais.

\section{REVISÃO TEÓRICA}

Especificamente, tem-se a eletricidade como uma forma de energia fundamental no consumo energético mundial, no entanto, os impactos causados por esse tipo de energia são muito expressivos e interferem no meio ambiente de várias maneiras: causam impactos nas funções dos ecossistemas incluindo a regulação climática, ciclagem de nutrientes, distribuição de água, dinâmica dos solos, dinâmica da população natural, entre outros (ECOINVENT CENTRE, 2010).

A matriz elétrica brasileira é composta em maior parte, por energia proveniente de fontes de origem renovável, predominantemente pela hidroeletricidade com $68,1 \%$, seguindo pelas termelétricas de gás natural com $9,1 \%$, as plantas de cogeração a biomassa com $8,2 \%$, as usinas nucleares com $2,6 \%$, as termelétricas que usam derivados de petróleo $2,4 \%$ e as com o carvão e seus derivados representa $4,2 \%$. A geração eólica está em um processo de desenvolvimentos e representa $5,4 \%$ da matriz elétrica brasileira (EPE, 2017).

O setor industrial é o maior consumidor de energia elétrica, com $37,6 \%$ do total utilizado no país, seguido pelo setor residencial e comercial, com respectivamente $25,6 \%$ e $17,2 \%$ do consumo do país. Já o setor de transporte é o que menos utiliza energia elétrica, com $0,4 \%$ do consumo total. Isto indica um uso potencial da eletricidade no setor de transporte, sempre que seu custo de geração seja competitivo e que tenha um menor impacto comparado com outras tecnologias. Além disso, deve ser considerada a origem (renovabilidade e sustentabilidade) e o incremento da capacidade de geração de eletricidade excedente que teria de ser produzido para suprir a nova demanda (EPE, 2017). Diante dessa realidade e com perspectivas de menores impactos ambientais e soluções para problemas energéticos, os veículos elétricos e híbridos e híbridos plug-ins estão ressurgindo nas frotas de automóveis ao redor do mundo.

\section{Veículo Elétrico}

O veículo elétrico não possui motor de combustão interna. Ele é movido por energia elétrica, seja provida por bateria, por célula de combustível, por placas fotovoltaicas (energia solar) ou ligados à rede elétrica, como os trólebus. Entre esses, grande parte das montadoras tem se concentrado no aperfeiçoamento e produção de veículo elétrico que utiliza bateria (BEV) (CASTRO et al., 2010).

Diante da característica de não realizar o processo de combustão para o uso final, esse tipo de veículo pode propiciar ganhos substanciais em termos de emissões de poluentes atmosféricos, já que ao menos no local onde é utilizado, não há emissões de substâncias nocivas ao meio ambiente, pois as emissões de escape são nulas. Outra vantagem do veículo elétrico é ser pouco ruidoso, tornando-o cada vez mais atrativo, principalmente para ser utilizado em grandes centros urbanos (LORA, 2002). 


\section{Veículos Híbridos Plug-in (PHEV)}

O veículo híbrido é caracterizado pelo uso de duas fontes distintas de energia para a sua movimentação: um motor elétrico e uma unidade de força auxiliar, além de um dispositivo para armazenar energia (BITSCHE et al., 2004). Uma das formas mais empregada e estudada de veículo híbrido é a que combina motor de combustão interna e motor elétrico.

Com os diferentes modos de operação um veículo híbrido torna-se muito mais flexível do que um veículo convencional. Com a configuração adequada e controle específico para cada condição de operação, este veículo pode aperfeiçoar o desempenho global, a eficiência e o nível de emissões poluentes (EHSANI et al., 2009). Estas combinações permitem que o motor térmico funcione em regime aproximadamente permanente, o que resulta em um mínimo de emissões e de consumo de combustível (KHEIR et al., 2004).

Um veículo híbrido "plug-in" (PHEV) é um híbrido cuja bateria utilizada para alimentar o motor elétrico pode ser carregada diretamente por meio de uma tomada. Esta característica permite combinar energia dos combustíveis fósseis com uma variedade de fontes de energia elétrica. Esse sistema causa impactos importantes, geralmente benéficos, sobretudo no consumo de petróleo e emissões de $\mathrm{CO}_{2}$ (LUKIC et al., 2008).

Um dos principais benefícios do PHEV deriva do fato de que as concessionárias de energia elétrica operam na maior parte do tempo em um nível de subutilização, devido às características do sistema energético para atender uma expectativa de pico de demanda. Assim, exceto por esses períodos de pico de uso, o sistema poderia gerar e fornecer uma quantidade substancial de energia necessária para abastecer os veículos que consomem eletricidade da rede a um baixo custo marginal de geração de energia (SOVACOOLet al., 2009).

\section{Análise do Ciclo de Vida}

A ACV é uma ferramenta utilizada para avaliar aspectos ambientais e energéticos associado a bens e processos durante o ciclo de vida, ou seja, do berço ao túmulo (proveniente da expressão inglês "from cradle to grave"). O ciclo inicia-se quando são requeridos os recursos da natureza, sejam materiais ou energéticos, para a manufatura de um determinado produto ou processo e finaliza-se após o cumprimento de sua função, retornando ao meio ambiente. A Figura 1 ilustra os estágios típicos do ciclo de vida de um produto (ROCHA et al., 2014; RUBIN; DAVIDSON, 2001).

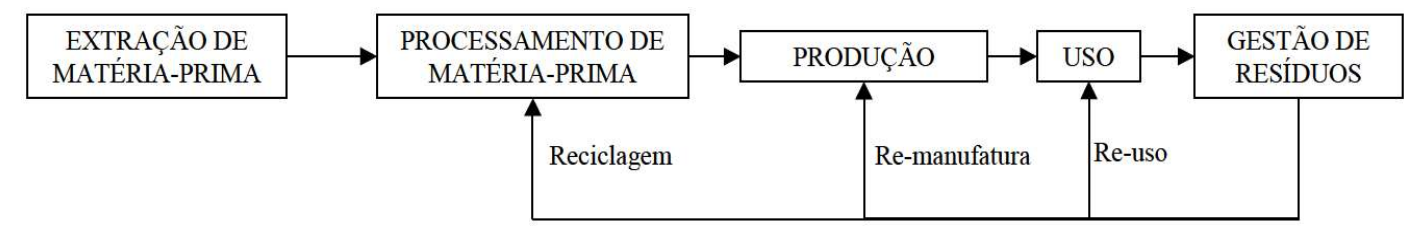

Figura 1: Etapas do Ciclo de Vida. Fonte: Rubin et al. (2001).

O processo para a análise do ciclo de vida geralmente compreende uma estrutura metodológica normatizada internacionalmente pela ISO, em que são determinadas as fases e os procedimentos gerais da 
execução de um estudo de ACV (FINKBEINER et al., 2006). De acordo com a norma ISO 14044 (ISO, 2006b) existem quatro fases de estudo para a ACV:

I- Definição do objetivo e escopo: O objetivo e o escopo são as primeiras fases para a realização da ACV. Estas etapas devem declarar de maneira inequívoca a aplicação pretendida e o processo de revisão crítica que se pretende adotar. No escopo deve-se considerar os limites temporal, tecnológicos e as fronteiras do sistema, relacionados aos temas.

II- Análise do inventário: A etapa da Análise do Inventário do Ciclo de vida envolve a coleta dos dados e os procedimentos utilizados para quantificar as entradas (materiais e energéticas) e as saídas (na forma emissões atmosféricas, efluentes líquidos e resíduos sólidos) ao longo do ciclo de vida do produto.

III- Avaliação do impacto do ciclo de vida: A avaliação de impactos do ciclo de vida tem como propósito determinar os potenciais de impactos ambientais das informações levantadas e quantificadas no inventário, além de fornecer subsídios para a etapa de interpretação.

IV- Interpretação dos resultados: $\mathrm{O}$ objetivo dessa etapa é combinar, resumir e discutir os resultados do estudo, com a finalidade de obter fundamentos para conclusões e recomendações que satisfaçam os objetivos iniciais. Esses resultados devem ser apresentados na forma de um relatório consistente.

\section{METODOLOGIA}

A estrutura metodológica deste trabalho se baseia normas padronizadas pelo ISO 14040 (ISO, 2006a) e ISO 14044 (ISO, 2006b). Para a análise dos impactos ambientais de sistemas de automóveis é necessária uma abordagem completa da ACV, que implica análise de materiais, energia e as emissões de um produto em seu ciclo de vida completo, desde que seja produzido, utilizado, até o seu fim de vida, que inclui o seu processo de reciclagem. Portanto, será realizado o estudo completo da ACV para dois sistemas distintos: (a) ciclo de vida do veículo, que inclui: montagem de veículos, manutenção, desmontagem e reciclagem. (B) um ciclo de vida de combustível que também referido como o well-to-whels (WTW), que inclui os seguintes passos: extração do combustível, produção, distribuição, armazenamento e utilização.

O objetivo deste trabalho é a realização de um estudo de avaliação dos impactos ambientais através da aplicação da ACV dos seguintes sistemas veículos/combustíveis: veículo com motor de combustão interna que utiliza a gasolina como fonte de energia (ICEVg); veículo elétrico movido a eletricidade (BEV); veículo híbrido plug-in movido a gasolina e eletricidade (PHEV), em condições brasileiras.

A função do sistema é a utilização de um meio de transporte para produzir o deslocamento de um passageiro por quilômetro. A unidade funcional estabelecida para o estudo é de $1 \mathrm{~km}$ percorrido pelo automóvel.

A abrangência da tecnologia considera veículos que utilizam motores de combustão interna de ignição por centelha, veículos elétricos e os híbridos. A abrangência temporal está associada ao uso final, ou seja, o momento em que se realizou o estudo (principais dados serão relativos ao ano de 2013). 
Fronteiras do sistema para o Cenário 1: ICEVg

O primeiro cenário em estudo representa um veículo com motor de combustão interna que utiliza a gasolina como combustível. Na configuração deste cenário, assume-se que a gasolina é o combustível convencional utilizado no Brasil. No entanto, devido a especificações e proporções definidas pela legislação em vigor no ano de 2013 , definiu-se que a gasolina distribuída ao usuário final, deve ser uma mistura de gasolina A (gasolina produzida pelas refinarias de petróleo e entregue diretamente às companhias distribuidoras), com $25 \%$ de álcool etílico anidro. A Figura 2 representa as fronteiras do sistema para este primeiro cenário.

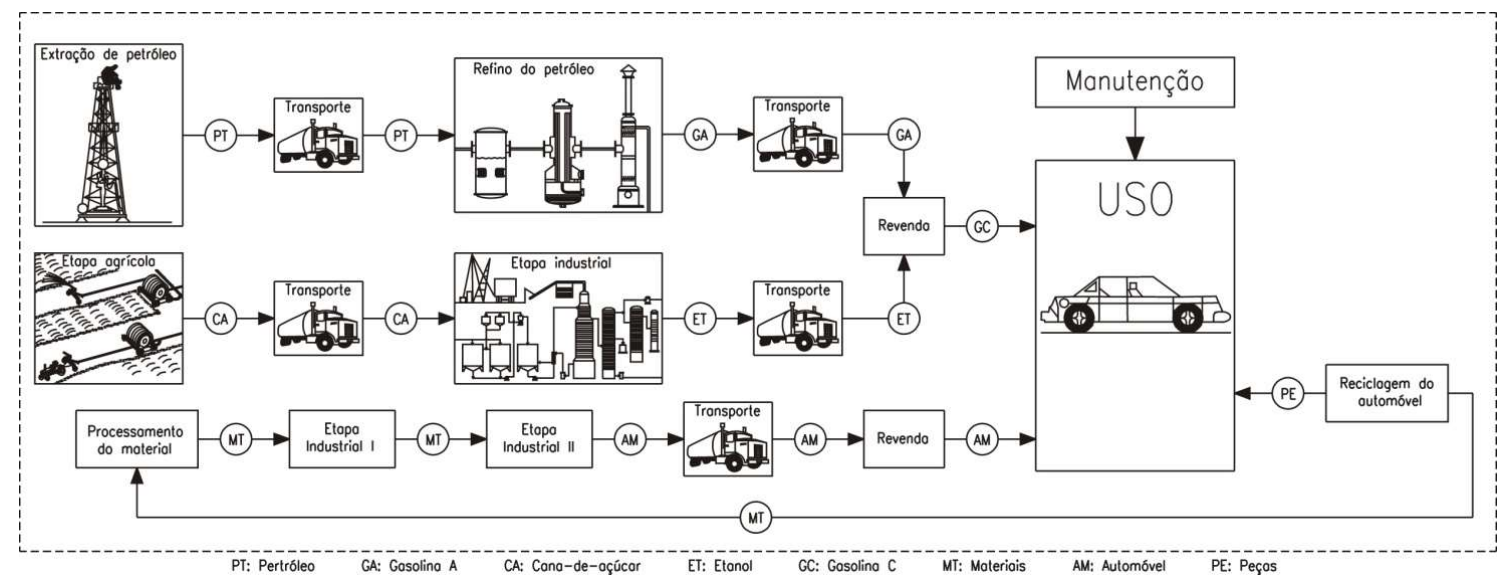

Figura 2: Fronteira do sistema para o ICEVg.

Fronteiras do sistema para o Cenário 2: PHEV

O segundo cenário em estudo é representado pelo veículo híbrido "plug-in" que além de possuir um motor elétrico, apresenta um motor de apoio, que no caso é o de combustão interna. A Figura 3 apresenta as fronteiras para este cenário.

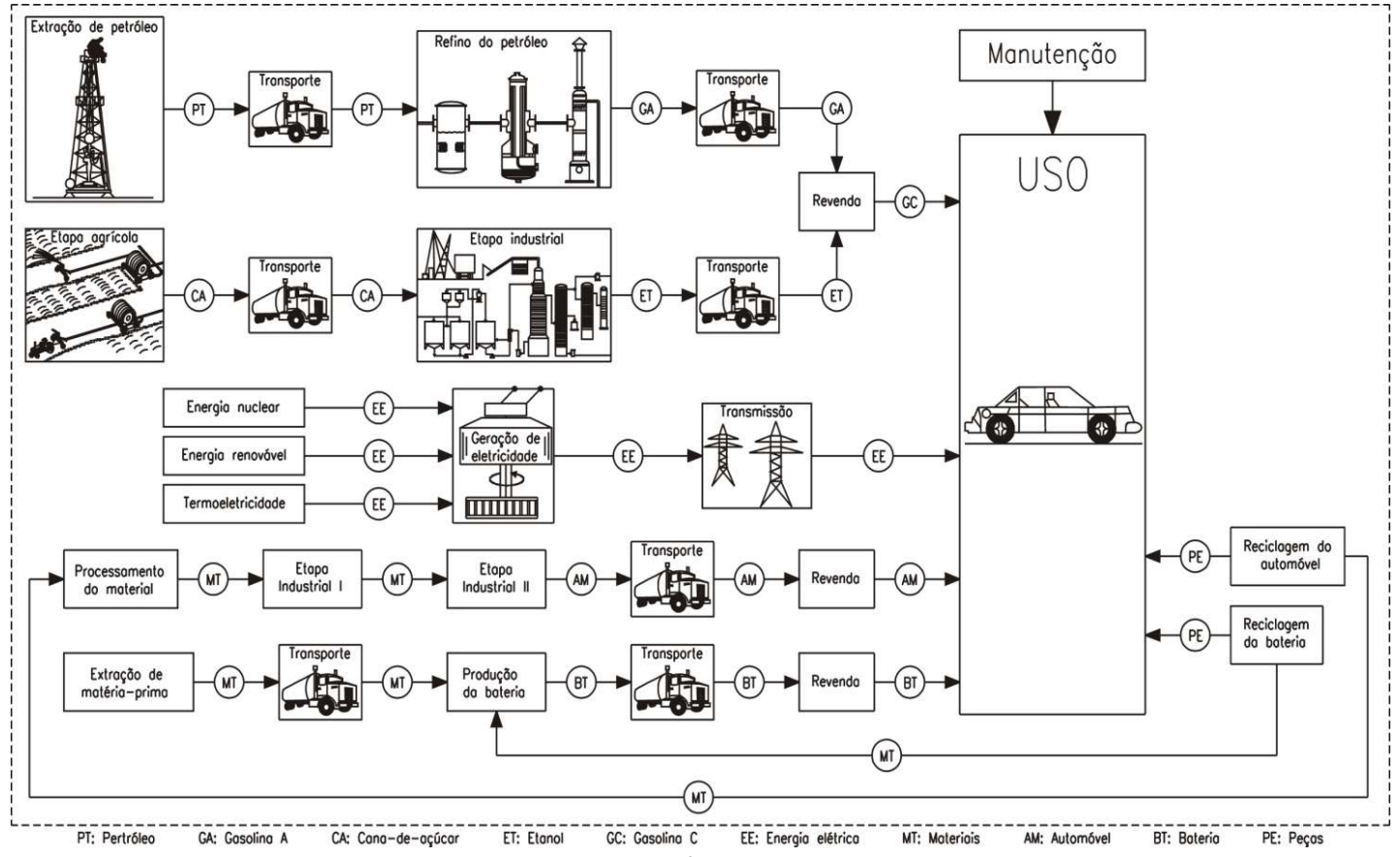

Figura 3: Fronteira do sistema para PHEV. 


\section{Fronteiras do sistema para o Cenário 3: BEV}

Este tipo de automóvel utiliza como sistema de propulsão o motor elétrico, um sistema de armazenamento de energia a bateria e como fonte de energia, a eletricidade. A Figura 4 apresenta as fronteiras para este cenário.

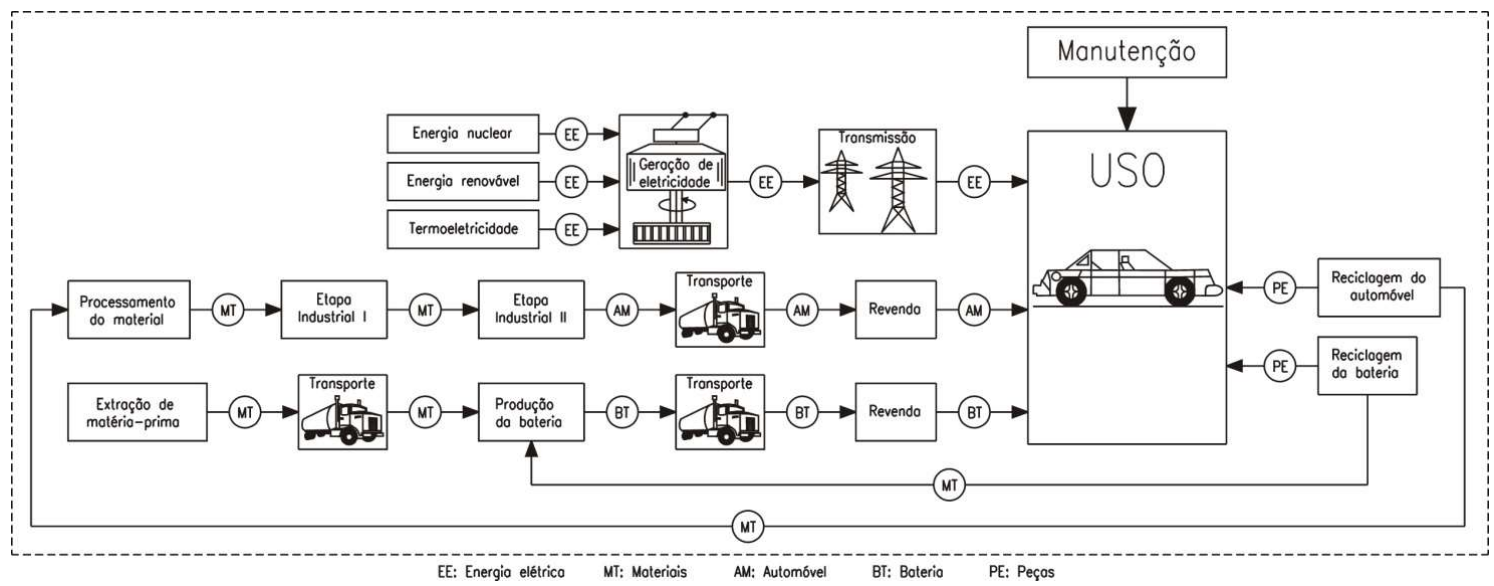

Figura 4: Fronteira do sistema para BEV.

\section{Análise do Inventário}

A etapa da Análise do Inventário do Ciclo de vida envolve a coleta dos dados e os procedimentos utilizados para quantificar as entradas e as saídas do estudo. A coleta de dados realizada nesse estudo foi dividida de acordo com cada sistema em estudo. A seguir são apresentadas as principais fontes de dados para cada sistema. Para os dados não inventariados, recorreu-se a base de dados do SimaPro.

\section{Etapa de Produção: Produção da Gasolina}

As etapas incluídas para o inventário da gasolina foram: extração e transporte do petróleo, refino, transporte da gasolina e uso. Isto inclui a pré-produção, correntes de fabricação do produto final, mas não foram inclusos a produção de máquinas utilizadas para essa funcionalidade.

Para o presente estudo, considerou-se que o petróleo utilizado para a produção da gasolina no Brasil possui duas origens distintas, sendo fixado o valor de $50 \%$ do petróleo proveniente de fontes internacionais, e $50 \%$ do petróleo proveniente de bacias nacionais. A Tabela 1 apresenta os principais materiais para a produção de 1 kg gasolina.

Tabela 1: Produção de gasolina.

\begin{tabular}{c|c}
\hline Material & Quantidade \\
\hline Petróleo $[\mathrm{kg}]$ & $1,11 \mathrm{E}+00$ \\
Gás natural $[\mathrm{kg}]$ & $3,93 \mathrm{E}-02$ \\
Água $[\mathrm{kg}]$ & $6,49 \mathrm{E}-03$ \\
Bauxita $[\mathrm{kg}]$ & $2,83 \mathrm{E}-04$ \\
Energia não especificada $[\mathrm{MJ}]$ & $3,28 \mathrm{E}+00$ \\
Uso do solo $\left[\mathrm{mm}^{2}\right]$ & $1,41 \mathrm{E}+01$ \\
\hline
\end{tabular}

Fonte: Borges (2004) 


\section{Etapa de Produção: Produção do etanol}

As etapas incluídas para o inventário do etanol foram: produção agrícola, transportes, produção de etanol, e seu uso final. Os dados utilizados dos inventários do etanol foram coletados de fontes secundárias. Outras variedades de dados foram obtidas a partir do banco de dados do software SimaPro. A Tabela 2 a seguir, apresenta os principais produtos para a produção de etanol, a partir de 1000 kg de cana-de-açúcar.

Tabela 2: Produção de Etanol.

\begin{tabular}{c|c}
\hline Material & Quantidade \\
\hline Cana de açúcar $[\mathrm{kg}]$ & 1000,0 \\
Água $\left[\mathrm{m}^{3}\right]$ & 1,0 \\
Ácido sulfúrico $[\mathrm{g}]$ & 520,0 \\
Cal $[\mathrm{kg}]$ & 0,81 \\
Produtos químicos $[\mathrm{g}]$ & 7,87 \\
Lubrificantes $[\mathrm{kg}]$ & 0,013 \\
Equipamentos $[\mathrm{kg}]$ & 0,047 \\
\hline
\end{tabular}

Fonte: Cavalett et al. (2013).

\section{Etapa de Produção: Produção da Eletricidade}

Para determinar o impacto da produção e uso da energia elétrica sobre o meio ambiente é necessário abordar os diferentes processos de conversão de energia que integram a matriz elétrica brasileira (MIRANDA, 2012). A Tabela 3 a seguir, apresenta a estrutura da oferta interna de eletricidade no Brasil, para o ano de 2013.

Tabela 3: Eletricidade por fonte de energia.

\begin{tabular}{|c|c|}
\hline Fonte de energia & Percentual (\%) \\
\hline Eletricidade proveniente de biomassa & 7,6 \\
\hline Eletricidade proveniente de carvão betuminoso & 2,6 \\
\hline Eletricidade proveniente do gás natural & 11,3 \\
\hline Eletricidade hidráulica & 70,6 \\
\hline Eletricidade eólica & 1,1 \\
\hline Eletricidade proveniente do diesel & 4,4 \\
\hline Eletricidade nuclear & 2,2 \\
\hline
\end{tabular}

Fonte: EPE (2014).

De uma maneira geral, tecnologias que utilizam combustíveis fósseis para a geração da eletricidade estão relacionadas com emissões diretas de GEE, principalmente durante a fase de operação da usina, enquanto que as tecnologias que utilizam combustíveis renováveis, grande parte das emissões ocorrem na fase de construção das estruturas e de equipamentos (WEISSER, 2007).

\section{Etapa de Produção: Produção do Automóvel}

O inventário do veículo Golf foi utilizado para este estudo. Os dados foram adaptados para modelar suas características específicas, principalmente os dados relacionados à carroceria e outras partes que são comuns ao segmento de carros de família. Os dados utilizados para o inventário de ciclo de vida do automóvel foram adaptados do estudo de Schweimer e Levin (2000), que teve como referência a planta de produção da Volkswagen, na Alemanha. Ainda que estes dados não sejam atuais, muitos autores ainda os utilizam, como por exemplo, Messagie et al. (2014); Faria et al. (2013) e Boureima et al. (2009), dentre outros. Isso de deve 
ao fato da confidencialidade de dados relativos à indústria automobilística, o que impede fontes de dados mais recentes e aprofundadas. A Tabela 4 apresenta os principais materiais utilizados para a produção dos materiais e montagem de 1 automóvel.

Tabela 4: Material para a produção de 1 automóvel.

\begin{tabular}{c|c}
\hline Material & Quantidade \\
\hline Bauxita $[\mathrm{kg}]$ & 25 \\
Chumbo $[\mathrm{kg}]$ & 740 \\
Cromo $[\mathrm{kg}]$ & 6 \\
Ferro $[\mathrm{kg}]$ & 1476 \\
Cobre $[\mathrm{kg}]$ & 78 \\
Níquel $[\mathrm{kg}]$ & 2 \\
Platina [kg] & 2000 \\
Zinco [kg] & 745 \\
Calcário $[\mathrm{kg}]$ & 666 \\
Sal gema $[\mathrm{kg}]$ & 100 \\
Água [m3] & 57 \\
Energia primária [GJ] & 86 \\
\hline
\end{tabular}

Fonte: Schweimer e Levin (2000).

\section{Etapa de produção: Produção da bateria de íon de lítio para BEV e PHEV}

Para o modelo representativo do automóvel de combustão interna, utilizou-se o veículo Golf. A fim de realizar uma comparação com o foco na produção e uso da bateria para veículos elétricos, optou-se por manter o inventário do automóvel, adicionando o ciclo de vida de uma bateria de íon de lítio.

De acordo com referências consultadas e a fim de simplificar os inventários, considerou-se a mesma bateria representante tanto a tecnologia PHEV como BEV. Na prática as características dessas baterias apresentam propriedades eletroquímicas e materiais um pouco diferentes, no entanto, Ying et al. (2006); Schexnayder et al. (2001); Gaines e Cuenca (2000) e Singh et al. (1998) combinaram os inventários, de modo a conseguir um inventário geral representativo para as duas tecnologias. A Tabela 5 apresenta os materiais necessários para a produção de uma bateria de íon de lítio.

Tabela 5: Materiais para a produção de 1 kg de bateria de íon de lítio.

\begin{tabular}{c|c}
\hline Material & Quantidade \\
\hline Pasta do eletrodo positivo [kg] & $2.50 \mathrm{E}-01$ \\
Pasta do eletrodo negativo [kg] & $8,00 \mathrm{E}-02$ \\
Substrato do eletrodo negativo [kg] & $3,60 \mathrm{E}-02$ \\
Substrato do eletrodo positivo [kg] & $8,30 \mathrm{E}-06$ \\
Eletrólito [kg] & $1,20 \mathrm{E}-01$ \\
Separador [kg] & $3,30 \mathrm{E}-02$ \\
Recipiente [kg] & $2,00 \mathrm{E}-01$ \\
Embalagem [kg] & $1,70 \mathrm{E}-01$ \\
Sistema de gestão da bateria [kg] & $2,00 \mathrm{E}-02$ \\
Eletricidade [MJ] & $2,70 \mathrm{E} 01$ \\
Óleo combustível [MW] & $2,90 \mathrm{E} 00$ \\
Gás natural [KW] & $2,20 \mathrm{E} 01$ \\
\hline
\end{tabular}

Fonte: Majeau-Bettez et al. (2011).

\section{Etapa de Produção: Etapa de Uso Final}

A distância adotada para o uso final dos veículos em estudo foi baseada na vida média útil de um automóvel no Brasil, ou seja, 160.000 km (WILLS, 2008). A mistura de gasolina e etanol é utilizada por 2 cenários distintos: ICEVg e PHEV. Para que se obtenham os dados necessários para o consumo dos 
automóveis, é necessário que se conheça o consumo $(\mathrm{km} / \mathrm{l})$ dos veículos e as proporções dos combustíveis utilizados. A Tabela 6 apresenta os dados utilizados para os inventários do uso ICEVg.

Tabela 6: Uso final do ICEVg.

\begin{tabular}{c|c}
\hline ICEVg & Quantidade \\
\hline Vida útil do veículo $(\mathrm{km})$ & 160.000 \\
Consumo específico $(\mathrm{km} / \mathrm{I})$ & 12,2 \\
Total de gasolina A (I) & 9836,1 \\
Total de etanol (I) & 3278,7 \\
\hline
\end{tabular}

O uso final para o BEV e para a parcela elétrica do PHEV consiste no mecanismo de carregar e descarregar a bateria. Essa grandeza está ligada diretamente à distância percorrida pelo veículo e perdas. Todos os dados relacionados ao consumo de energia de operação do BEV e do PHEV e outras perdas adicionais foram estimados em veículos existentes e considerações teóricas (FARIA et al., 2013). A Tabela 7 contém os dados relativos aos consumos do PHEV e do BEV.

Tabela 7: Uso final BEV e PHEV.

\begin{tabular}{c|c|c}
\hline Características do automóvel & PHEV & BEV \\
\hline Peso da bateria $(\mathrm{kg})$ & $197,0^{\mathrm{a}}$ & $300^{\mathrm{a}}$ \\
Peso do automóvel & $1715,0^{\mathrm{a}}$ & $1521^{\mathrm{a}}$ \\
Alcance $(\mathrm{km})$ & $580(80 \mathrm{ED}+500 \mathrm{ER})^{\mathrm{a}}$ & $160^{\mathrm{a}}$ \\
Consumo eletricidade $(\mathrm{kWh} / 100 \mathrm{~km})$ & $17^{\mathrm{a}}$ & $17^{\mathrm{a}}$ \\
Consumo gasolina $(\mathrm{l} / 100 \mathrm{~km})$ & $0(\mathrm{ED}) 6.9(\mathrm{GD}) 3.9(\mathrm{MD})^{\mathrm{a}}$ & $0^{\mathrm{a}}$ \\
Distância modo eletricidade $(\mathrm{km})$ & 22400,0 & 160000 \\
Distância modo gasolina $(\mathrm{km})$ & 137600 & 0 \\
Consumo eletricidade total $(\mathrm{kWh})$ & 3808 & 27200 \\
Consumo gasolina A total $(\mathrm{I})$ & 7120,8 & 0 \\
Consumo de etanol total (I) & 2373,6 & 0 \\
\hline
\end{tabular}

Fonte: Faria et al. (2013). Legenda: ED: Modo elétrico; ER: Modo gasolina; ED: Apenas elétrico; GD: Apenas gasolina; MD: Unidade mista.

\section{Etapa de Produção: Etapa de Fim de Vida}

A etapa de fim de vida desse estudo considera apenas as reciclagens do automóvel e da bateria de íon de lítio. Para este trabalho, o fim-de-vida foi modelado com relação ao estado-da-arte das instalações de reciclagem belgas devido à disponibilidade de dados. Adotou-se um consumo de energia geral para todo o processo de reciclagem de $66 \mathrm{kWh} / \mathrm{t}$.

A recuperação e reciclagem de materiais da bateria tem aumentado significativamente devido principalmente aos custos elevados das matérias-primas para a produção, e em alguns países devido ao rigor da legislação (GAO; WINFIELD, 2012). No entanto, a reciclagem da bateria de íon de lítio raramente é incluída nas avaliações energéticas e ambientais, devido ao pouco enfoque que essa etapa possui atualmente, pois esses processos ainda estão em fase de experimento. Não é como o caso da reciclagem da bateria de chumbo ácido, em que esse processo de reciclagem já é consolidado.

Um aspecto considerável a respeito da reciclagem da bateria é a verificação da energia necessária para recuperar os materiais, pois se esse valor exceder a energia necessária para utilizar materiais virgens, a reciclagem torna-se menos atrativa (NOTTER et al., 2010). 
A Avaliação dos Impactos do Ciclo de Vida será realizada com o auxílio do software SimaPro, elaborado pela Pré-Consultants. Esse programa é utilizado com o intuito de analisar, identificar e comparar os impactos ambientais dos cenários analisados.

\section{Seleção e Definição dos Métodos e das Categorias do Impacto}

Este trabalho usou o método CML 2 baseline 2000 v.2.03 desenvolvido pelo Centro de Ciências Ambientais da Universidade de Leiden (CML). O método cobre todas as emissões e recursos relacionados aos impactos para os quais modelos de caracterização aceitáveis estão disponíveis. As categorias de impacto escolhidas para esse estudo foram: Potencial de Aquecimento Global, Toxicidade Humana, Acidificação. A justificativa é que tais categorias representam os principais impactos ambientais produzidos pelo setor automotivo.

O Potencial de aquecimento global é uma medida de como uma determinada quantidade de gás do efeito estufa contribui para o aquecimento global. O potencial de toxicidade humana abrange os impactos na saúde humana das substâncias tóxicas presentes no ambiente. O potencial de acidificação relaciona-se com os poluentes acidificantes que tem impactos no solo, água subterrânea, águas superficiais, organismos biológicos, ecossistemas e materiais (edifícios).

\section{RESULTADOS}

A avaliação dos impactos ambientais foi feita considerando os diferentes aspectos de cada produto, ou seja, well-to-wheels das fontes de energia e o ciclo de vida do veículo, e o ciclo de vida das baterias de íon de lítio, incluindo as principais entradas e saídas envolvidas nos processos.

\section{Potencial de Aquecimento Global}

Conforme se observa na Figura 5 o ICEVg possui o maior impacto para o Potencial de Aquecimento Global (GWP), com a emissão de 2,29E-01 kg de $\mathrm{CO}_{2}-$ eq. $/ \mathrm{km}$. Isso ocorre devido à grande quantidade de $\mathrm{CO}_{2}$ lançados na atmosfera a partir da combustão da gasolina. Esse índice é menor que as médias mundiais, devido principalmente à adição de etanol anidro à composição da gasolina A. A produção do automóvel para o ICEVg é responsável por cerca de $25 \%$ da emissão total de $\mathrm{CO}_{2}$-eq., que tem como maior contribuinte as etapas de processamentos dos materiais e manufatura. No entanto esse valor é relativamente baixo devido à utilização de energia elétrica de baixa emissão de $\mathrm{CO}_{2}$ utilizada nesses processos. A produção do combustível é responsável por $14 \%$ das emissões de $\mathrm{CO}_{2}$-eq. As emissões de escapamento são responsáveis por aproximadamente $60 \%$ do total das emissões de $\mathrm{CO}_{2}$-eq., e reciclagem do automóvel por $-4,0 \%$, esse valor negativo se deve ao fato da reciclagem do veículo contribuir com produtos evitados (produtos que não devem ser extraídos da natureza novamente).

O PHEV possui o segundo maior resultado para o GWP, com o valor de 1,84E-01 kg de $\mathrm{CO}_{2}-\mathrm{eq} . / \mathrm{km}$. Este resultado é variável conforme a matriz elétrica considerada e pelas quantidades específicas de gasolina e eletricidade adotadas, que neste caso a utilização do veículo em modo elétrico corresponde apenas a 7,5\% 
da quilometragem total percorrida. A produção do automóvel é responsável por aproximadamente $32 \%$ das emissões de $\mathrm{CO}_{2}$-eq., enquanto que para a produção dos combustíveis, cerca de $17 \%$. Já para as emissões de escape, o resultado foi de $36 \%$, considerado baixo, devido à característica de emissão de escape zero, quando o veículo utiliza o modo elétrico. A produção da bateria de íons de lítio assume uma participação aproximada de $14 \%$ das emissões de $\mathrm{CO}_{2}$-eq. Esse resultado é inferior ao da produção da bateria de íon de lítio para $\mathrm{BEV}$, devido ao menor peso da bateria. A reciclagem do automóvel é responsável $-4,5 \%$ das emissões de $\mathrm{CO}_{2}$-eq., enquanto que da bateria aproximadamente $-3,0 \%$.

Com o resultado de $1,18 \mathrm{E}-01 \mathrm{~kg}$ de $\mathrm{CO}_{2}-\mathrm{eq} . / \mathrm{km}$, o melhor resultado foi para o $\mathrm{BEV}$, que possui como característica emissões de escape zero. Um fator determinante para este resultado foi à utilização de dados referentes à matriz elétrica brasileira, que possui uma base energética renovável, consequentemente uma menor emissão de gases do efeito estufa na etapa de produção da energia. A etapa de produção da bateria de íon de lítio corresponde a 35\% das emissões de $\mathrm{CO}_{2}$-eq. A produção do automóvel é responsável por cerca de $50 \%$ da emissão total de $\mathrm{CO}_{2}$-eq., enquanto que a produção do combustível por aproximadamente $15 \%$. A reciclagem do automóvel é responsável por cerca de $-5,5 \%$ e a reciclagem da bateria por $-3,5 \%$.

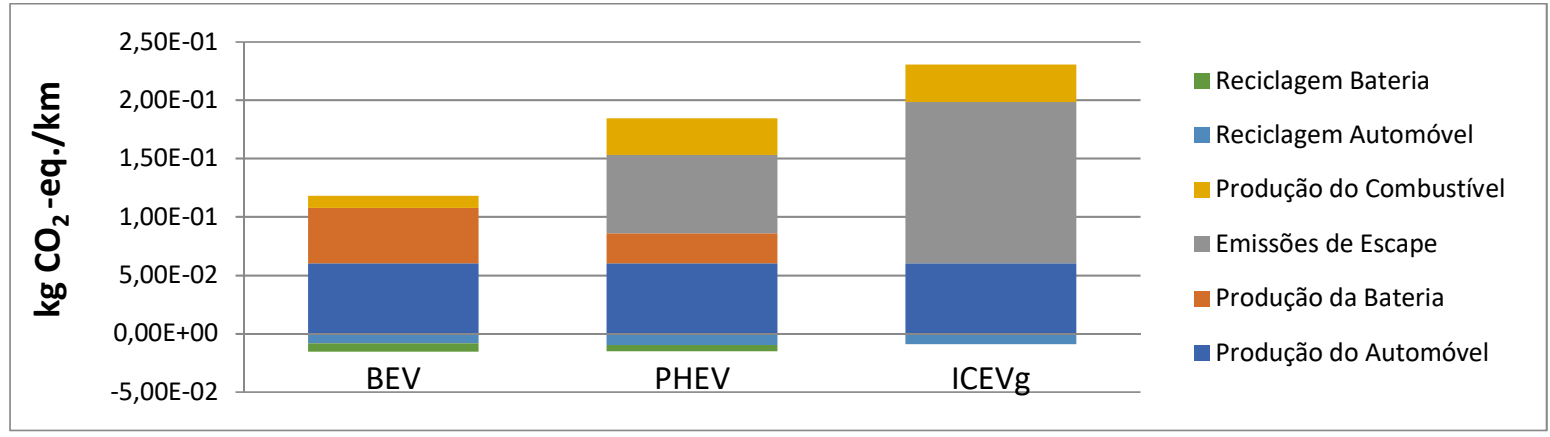

Figura 5: Potencial de aquecimento global.

\section{Potencial de Acidificação}

De acordo com a Figura 6, o ICEVg possui o maior potencial de acidificação, com 5,86E- 04 kg SO ${ }_{2}-$ eq./km. O maior responsável por esse resultado é o etanol anidro adicionado à gasolina. A produção do combustível é a etapa com participação mais significativa para esta categoria de impacto, que contribui com aproximadamente $72 \%$ das emissões. A produção do automóvel é a responsável por cerca de $20 \%$ das emissões $\mathrm{SO}_{2}$ eq. As emissões de escape são responsáveis por $8 \%$, e a reciclagem do automóvel por $-4 \%$ das emissões de $\mathrm{SO}_{2}$-eq.

O segundo maior potencial é para o PHEV com o resultado de 5,19E-04 kg SO${ }_{2}$-eq. $/ \mathrm{km}$. A utilização de energia elétrica proveniente de fontes renováveis beneficia o resultado para essa categoria de impacto. A produção da bateria de íon de lítio não tem uma participação significativa para esta categoria de impacto, com apenas $4 \%$ do total de $\mathrm{SO}_{2}$-eq total. A produção do automóvel é a responsável por cerca de $24 \%$ das emissões $\mathrm{SO}_{2}$-eq. A produção do combustível é a etapa com participação mais significativa para esta categoria de impacto, que contribui com aproximadamente $66 \%$ das emissões. As emissões de escape são responsáveis por $6 \%$, e a reciclagem do automóvel $-4 \%$ e da bateria por $-1 \%$ das emissões de $\mathrm{SO}_{2}$-eq. 
O melhor resultado foi para $\mathrm{BEV}$, com o valor de 3,03E-04 kg SO${ }_{2}-\mathrm{eq} . / \mathrm{km}$. A produção da bateria de íon de lítio possui uma participação mais significativa que a para o PHEV, devido à massa dessa bateria, com $10 \%$ da contribuição total de $\mathrm{SO}_{2}$-eq. As atividades relacionadas à mineração e metalurgia, necessárias para a produção do níquel nos eletrodos e nos coletores de corrente das baterias de íon de lítio são as etapas que mais contribuem para essa categoria de impacto. A produção do automóvel é a responsável por cerca de $39 \%$ das emissões $\mathrm{SO}_{2}$-eq. A produção do combustível contribui com aproximadamente $49 \%$ das emissões. As emissões de escape por $6 \%$, e a reciclagem do automóvel é responsável por $-8 \%$ e da bateria por $-2 \%$ das emissões de $\mathrm{SO}_{2}$-eq.

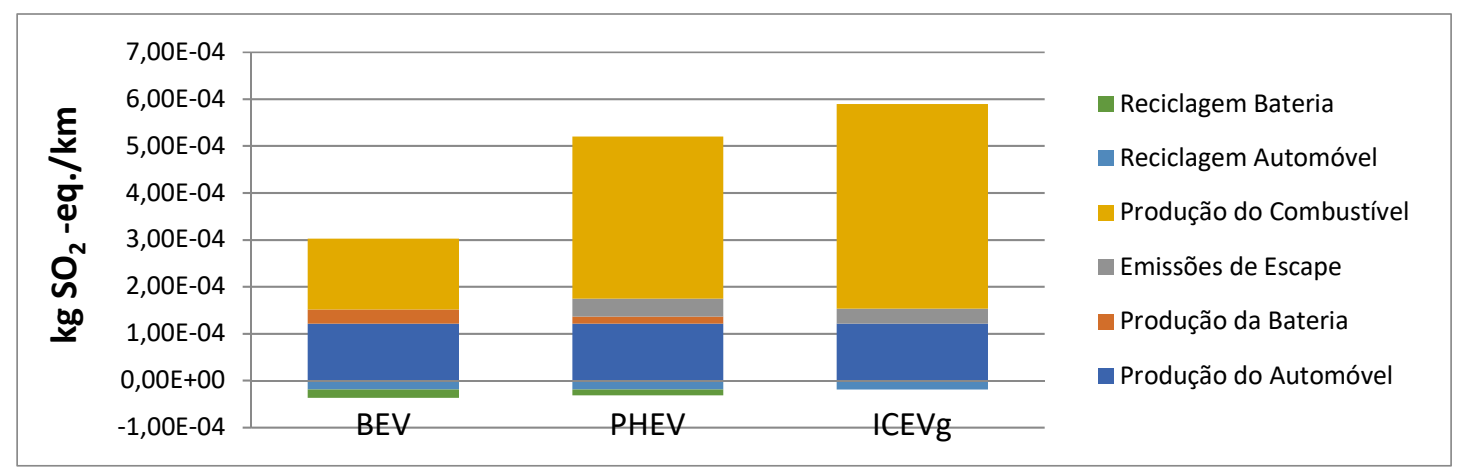

Figura 6: Potencial de acidificação.

\section{Potencial de Toxicidade Humana}

De acordo coma Figura 7 o maior potencial de toxicidade humana é para BEV, com 7,04E-02 kg 1,4 DB-eq./km (1,4-diclorobenzeno equivalentes). A produção da bateria de íon de lítio possui a participação mais significativa para esta categoria de impacto, com $57 \%$ da contribuição total de 1,4 DB-eq. Esse resultado deve-se principalmente aos elevados encargos das substâncias tóxicas que são liberadas por atividades de mineração de metais (como por exemplo, de níquel, cobre, platina e alumínio). A produção do automóvel é a responsável por aproximadamente $37 \%$ das emissões. A produção da eletricidade é a menor contribuinte para esta categoria de automóvel, com apenas $6 \%$ das emissões. A reciclagem do automóvel é responsável por $-5 \%$ e da bateria por $-6 \%$ das emissões de 1,4 DB-eq.

O PHEV possui o segundo maior resultado para a toxicidade humana com 6,38E-02 kg 1,4 DB-eq./km. A produção da bateria de íon de lítio é responsável por 30\% da contribuição total de 1,4 DB-eq. Esse valor é menor que a produção da bateria do BEV, devido à sua menor massa. A produção do automóvel é a responsável por aproximadamente $40 \%$ das emissões. A produção dos combustíveis corresponde a $29 \%$ dos impactos para a toxicidade humana. As emissões de escape são responsáveis por valores mínimos para o potencial de toxicidade humana. A reciclagem do automóvel é responsável por aproximadamente -6,5\% e da bateria por $-4 \%$ das emissões de 1,4 DB-eq.

O terceiro maior potencial de toxicidade humana é para ICEVg com o valor de 4,30E-02 kg 1,4 DBeq./km. A produção do automóvel é a responsável por aproximadamente $60 \%$ das emissões. A produção dos combustíveis corresponde a $38 \%$ dos impactos para a toxicidade humana. Esse resultado é decorrente principalmente de substâncias tóxicas utilizadas/emitidas no refino do petróleo. As emissões de escape 
correspondem a uma participação reduzida para a toxicidade humana. A reciclagem do automóvel é responsável por uma redução -8\% das emissões de 1,4 DB-eq.

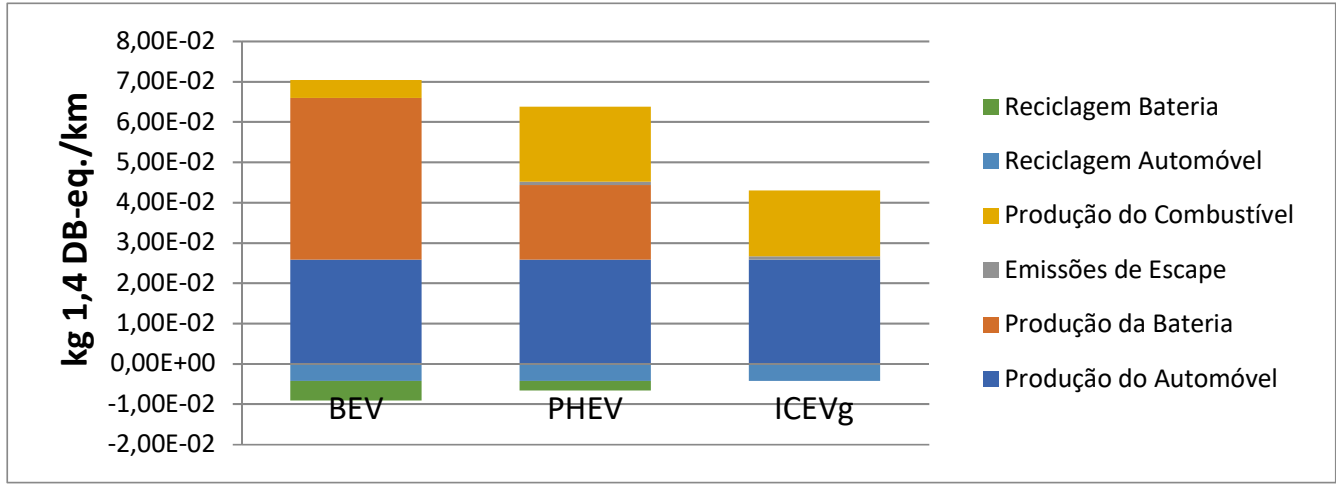

Figura 7: Potencial de toxicidade humana.

\section{Comparação Geral entre os Veículos}

De uma maneira geral, não existe uma tecnologia com um melhor desempenho em todas as categorias de impacto. No entanto, de acordo com a Figura 8, a partir de um indicador único, que seria calculado através da área de cada sistema veicular, o menor valor relativo seria para o BEV. Para os polos que apresentam as categorias acidificação e aquecimento global, os maiores valores são para os veículos que utilizam a gasolina como parte integrante de seu combustível. Para o polo que apresenta a categoria de toxicidade humana, os maiores valores são para os veículos que possuem a bateria de íon de lítio.

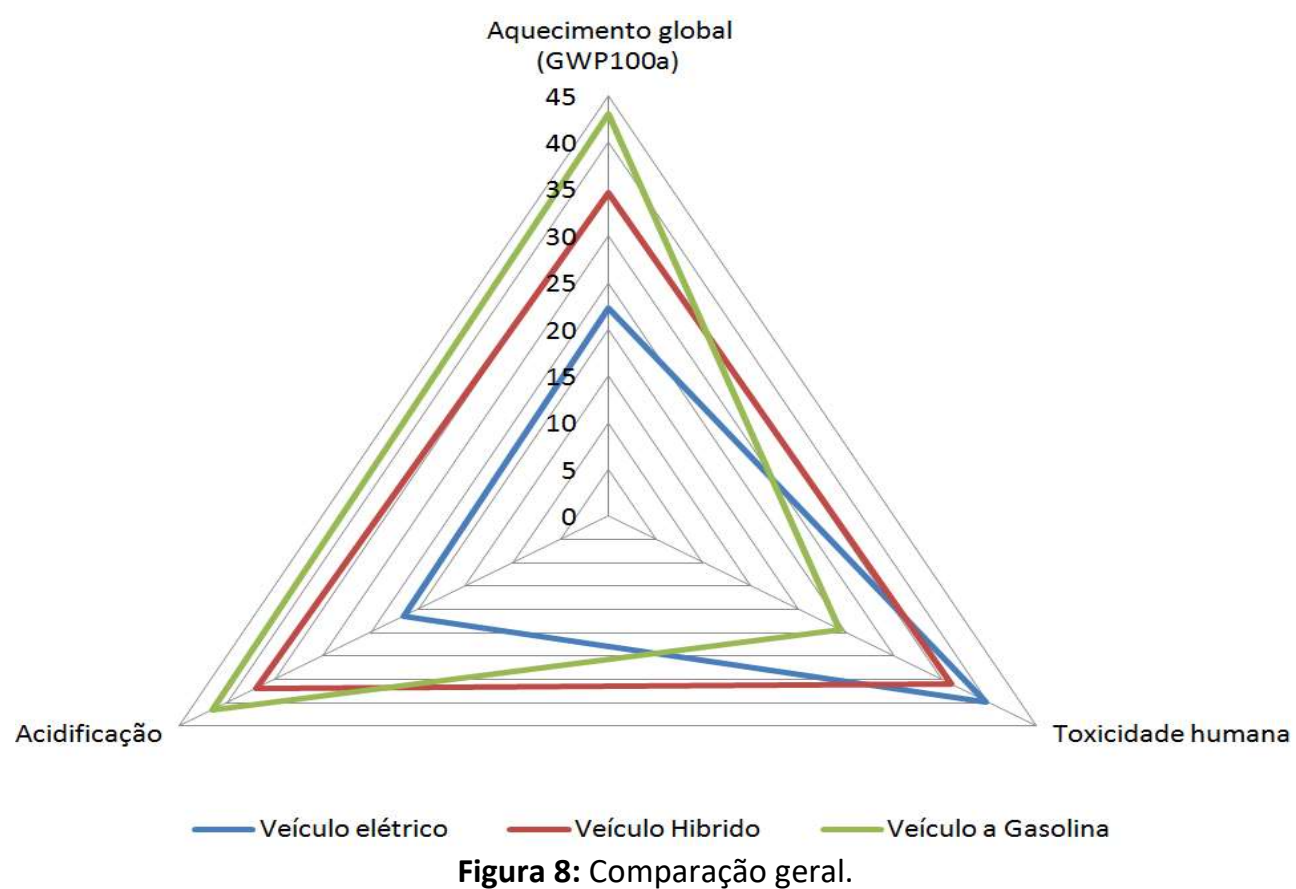

\section{CONCLUSÕES}

Os impactos ambientais quantificados a partir da aplicação da ACV para estudo foram: aquecimento global, eutrofização e toxicidade humana, e estes podem ser utilizados como uma ferramenta auxiliar em tomadas de decisão visando a busca pela sustentabilidade. No entanto, para uma conclusão precisa sobre 
qual o sistema automotivo mais adequado para o uso, deve-se realizar uma investigação holística a respeito da produção e uso do automóvel, sendo que outros parâmetros também devem ser investigados como, por exemplo, eficiência energética, uso do solo, custos, disponibilidade de materiais na natureza, rede de distribuição, logística das fontes de energias, estratégias governamentais, dentre outros.

De uma maneira geral, o PHEV apresenta características similares ao ICEVg, pois também utiliza a gasolina como combustível, mas devido ao uso de eletricidade, os resultados para o potencial de aquecimento global e acidificação são menores que o IVECg. O PHEV também apresenta características semelhantes ao BEV por fazer o uso da eletricidade e da bateria de íons de lítio, e esta, promove maiores resultados para o potencial de toxicidade humana.

\section{REFERÊNCIAS}

BALAT, M.; BALAT, H.; ÖZ, C.. Progress in bioethanol processing. Progress in Energy and Combustion Science, Oxford, v.34, n.5, p.551-573, 2008. DOI: http://doi.org/10.1016/j.pecs.2007.11.001

BITSCHE, O.; GUTMANN, G.. Systems for hybrid cars. Journal of Power Sources, Amsterdã, v.127, n.1-2, p.8-15, 2004. DOI: http://doi.org/10.1016/j.jpowsour.2003.09.003

BORGES, F.J.. Inventário do ciclo de vida do PVC produzido no Brasil. Dissertação (Mestrado em Engenharia Química) Universidade de São Paulo, São Paulo, 2004.

BOUREIMA, F.; MESSAGIE, M.; MATHEYS, J.; WYNEN, V.; SERGEANT, N.; VAN MIERLO, J.; DE VOS, M.; DE CAEVEL, B.. Comparative LCA of electric, hybrid, LPG and gasoline cars in a Belgian context. World Electric Vehicle Journal. Stavanger, v.3, p.0469-0476, 2009.

CASTRO, B.H.R; FERREIRA, T.T.. Veículos elétricos: aspectos básicos, perspectivas e oportunidade. BNDES Setorial, Rio de Janeiro, n.32, p.267-310, 2010.

CAVALETT, O.; CHAGAS, M.F.; SEABRA, J.E.A.; BONOMI, A.. Comparative LCA of ethanol versus gasoline in Brazil using different LCIA methods. The International Journal of Life Cycle Assessment, Heidelberg, v.18, n.3, p.647-658, 2013. DOI: http://doi.org/10.1007/s11367-012-0465-0

ECOINVENT CENTRE. Ecoinvent data and reports v.2.2. Dübendorf: Swiss Centre for Life Cycle Inventories, 2010.

EHSANI, M.; GAO, Y.; EMADI, A.. Modern electric, hybrid electric, and fuel cell vehicles: fundamentals, theory, and design. 2 ed. Nova lorque: CRC PRESS, 2009.

EPE. Empresa de Pesquisa Energética. Balanço Energético Nacional 2017: Ano base 2016. Rio de Janeiro: Empresa de Pesquisa Energética (EPE), 2017.

EPE. Empresa de Pesquisa Energética. Balanço Energético Nacional 2014: Ano base 2013. Rio de Janeiro: Empresa de Pesquisa Energética (EPE), 2014.

EPE. Empresa de Pesquisa Energética. Balanço Energético Nacional 2011: Ano base 2010. Rio de Janeiro: Empresa de Pesquisa Energética (EPE), 2011.
FARIA, R.; MARQUES, P.; MOURA, P.; FREIRE, F.; DELGADO, J.; ALMEIDA, A.T.. Impact of the electricity mix and use profile in the life-cycle assessment of electric vehicles. Renewable and Sustainable Energy Reviews, Oxford, v.24, p.271-2287, 2013. DOI: http://10.1016/j.rser.2013.03.063

FINKBEINER, M.; INABA, A.; TAN, R.B.H.; CHRISTIANSEN, K.; KLÜPPEL, H.-J.. The new international standards for life cycle assessment: ISO 14040 and ISO 14044. The International Journal of Life Cycle Assessment, Heidelberg, v.11, n.2, p.80-85, 2006. DOI: http://doi.org/10.1065/lca2006.02.002

GAINES, L.; CUENCA, R.. Costs of lithium-ion batteries for vehicles. Argonne, Illinois: Center for Transportation Research, Argonne National Laboratory, 2000.

GAO, L.; WINFIELD, Z.C.. Life cycle assessment of environmental and economic impacts of advanced vehicles. Energies, Basileia, v.5, n.3, p.605-620, 2012. DOI: http://doi.org/10.3390/en5030605

ISO. International Standards Organization. Environmental Management. Life Cycle Assessment. Principles and Framework. ISO 14040. Genebra: International Standards Organization, 2006a.

ISO. International Standards Organization. Environmental Management. Life Cycle Assessment. Requirements and Guidelines. ISO 14044. Genebra: International Standards Organization, 2006b.

KHEIR, N.A.; SALMAN, M.A.; SCHOUTEN, N.J.. Emissions and fuel economy trade-off for hybrid vehicles using fuzzy logic. Mathematics and Computers in Simulation, Amsterdã, v.66, n.2-3, p.155-172, 2004. DOI:

http://doi.org/10.1016/j.matcom.2003.11.007

LORA, E.E.S.. Prevenção e controle da poluição nos setores energético, industrial e de transporte. 2 ed. Rio de Janeiro: Editora Interciência, 2002.

LUKIC, S.M.; CAO, J.; BANSAL, R.C.; RODRIGUEZ, F.; EMADI, A.. Energy storage systems for automotive applications. IEEE Transactions on Industrial Electronics, Nova lorque, v.55, n.6, p.2258-2267, 2008. DOI: http://doi.org/10.1109/TIE.2008.918390 
MAJEAU-BETTEZ, G.; HAWKINS, T.R.; STR ØMMAN, A.H.. Life cycle environmental assessment of lithium-ion and nickel metal hydride batteries for plug-in hybrid and battery electric vehicles. Environmental Science \& Technology, Washington, v.45, n.10, p.4548-4554, 2011. DOI: http://doi.org/10.1021/es103607c

MESSAGIE, M.; BOUREIMA, F.-S.; COOSEMANS, T.; MACHARIS, C.; VAN MIERLO, J.. A range-based vehicle life cycle assessment incorporating variability in the environmental assessment of different vehicle technologies and fuels. Energies, Basileia, v.7, n.3, p.1467-1482, 2014. DOI: http://doi.org/10.3390/en7031467

MIRANDA, M.M.. Fator de emissão de gases de efeito estufa da geração de energia elétrica no Brasil: implicações da aplicação da avaliação do ciclo de vida. Dissertação (Ciências da Engenharia Ambiental) - Universidade de São Paulo, São Carlos, 2012.

MOMOH, O.D.; OMOIGUI, M.O.. An overview of hybrid electric vehicle technology. In: IEEE Vehicle Power and Propulsion Conference, 5. Anais. Dearborn, p.1286-1292, 2009.

NOTTER, D.A.; GAUCH, M.; WIDMER, R.; WÄGER, P.; STAMP, A.; ZAH, R.; ALTHAUS, H.-J.. Contribution of Li-ion batteries to the environmental impact of electric vehicles.

Environmental Science \& Technology, Washington, v.44, n.17, p.6550-6556, 2010. DOI: http://10.1021/es903729a

ROCHA, M.H. ; CAPAZ, R.S. ; LORA, E.E.S. ; NOGUEIRA, L.A.H. ; LEME, M.M.V. ; RENÓ, M.L.G. ; ALMAZÁN, O.O.. Life cycle assessment (LCA) for biofuels in Brazilian conditions: A meta-analysis. Renewable and Sustainable Energy Reviews, Oxford, v.37, p.435-459, 2014. DOI:

http://doi.org/10.1016/j.rser.2014.05.036

RUBIN, E.S.; DAVIDSON, C.I.. Introduction to engineering and the environment. 1 ed. Nova lorque: McGraw-Hil Higher Education, 2001.

SCHEXNAYDER, S.M.; DAS, S.; DHINGRA, R.; OVERLY, J.G.; TONN, B.E.; PERETZ, J.H.; WAIDLEY, G.; DAVIS, G.A.
Environmental evaluation of new generation vehicle and vehicle components. Oak Ridge: Oak Ridge National Laboratory (ORNL), 2001.

SCHWEIMER, G.W.; LEVIN, M.. Life cycle inventory for the Golf A4. Wolfsburg: Wolkswagen AG, Research, Environment and Transport, 2000.

SINGH, M.; CUENCA, R.; FORMENTO, J.; GAINES, L.; MARR, B.; SANTINI, D.; WANG, M.; ADELMAN, S.; KLINE, D.; MARK, J.; OHI, J.; RAU, N.; FREEMAN, S.; HUMPHREYS, K.; PLACET, $M$.. Total energy cycle assessment of electric and conventional vehicles: an energy and environmental analysis. Springfield: Argonne National Laboratory (ANL), National Renewable Energy Laboratory (NREL) e Pacific Northwest National Laboratory (PNNL), 1998.

SOVACOOL, B.K.; HIRSH, R.F.. Beyond batteries: An examination of the benefits and barriers to plug-in hybrid electric vehicles (PHEVs) and a vehicle-to-grid (V2G) transition. Energy Policy, Oxford, v.37, n.3, p.1095-1103, 2009. DOI: http://10.1016/j.enpol.2008.10.005

WEISSER, D.. A guide to life-cycle greenhouse gas (GHG) emissions from electric supply technologies. Energy, Oxford, v.32, n.9, p.1543-1559, 2007. DOI: http://doi.org/10.1016/j.energy.2007.01.008

WILLS, W.. 0 aumento da eficiência energética nos veículos leves e suas implicações nas emissões de gases de efeito estufa - Cenários brasileiros entre 2000 e 2030. Dissertação (Ciências em Planejamento Energético) - Universidade Federal do Rio de Janeiro, Rio de Janeiro, 2008.

XIMENES, A.; MERLI, A.G.; CAMPOS, E.M.A.; DIAS, J.V.P.P.. O impacto ambiental devido a política de crescimento da frota de veículos. Revista Ciências do Ambiente On-Line, Campinas, v.4, n.2, p.1-7, 2008.

YING, T.-K.; GAO, X.-P.; HU, W.-K.; WU, F.; NORÉUS, D.. Studies on rechargeable NiMH batteries. International Journal of Hydrogen Energy, Oxford, v.31, n.4, p.525-530, 2006. DOI: http://doi.org/10.1016/j.ijhydene.2005.04.018 